\title{
Análise das transformações metalúrgicas ocorridas na ZAC de aço inoxidável duplex UNS S31803 soldado pelo processo GMAW pulsado
}

\author{
Analysis of metallurgic transformations on \\ UNS S31803 duplex stainless steel HAZ \\ welded by pulsed GMAW process
}

\author{
William Haupt ${ }^{1}$, Kauê Correa Riffel ${ }^{1}$, \\ Claudia Fanezi ${ }^{2}$, Telmo Roberto Strohaecker ${ }^{2}$
}

\author{
${ }^{1}$ LAMP - Laboratório de Materiais e Processos, departamento de Engenharia Mecânica, prédio I2, Br 285, São José, \\ Passo Fundo - RS. \\ e-mail:williamhaupt@upf.br; kaueriffel@hotmail.com \\ ${ }^{2}$ LAMEF- Laboratório de Metalurgia Física, departamento de Engenharia Metalúrgica, prédio 43820, Av. Bento Gonçal- \\ ves, 9500, Porto alegre - RS. \\ e-mail:cfanezi@gmail.com; telmo@demet.ufrgs.br
}

\section{RESUMO}

Os aços inoxidáveis duplex (AID) são materiais que possuem excelente resistência mecânica e elevada resistência à corrosão em meios agressivos, fatores que contribuem para o seu emprego em larga escala nas indústrias de petróleo e de processos químicos. Estas propriedades são garantidas pela sua composição microestrutural com $50 \%$ de fase ferrita e $50 \%$ de fase austenita em média, garantida pelas adições de elementos de liga balanceados e pela temperatura de solubilização. Em muitas aplicações o processo de soldagem é utilizado, sendo realizados ciclos térmicos no material durante o processo, que geram modificações microestruturais. Se o processo não for devidamente controlado podem ocorrer severas modificações da microestrutura característica do material e ainda causar precipitação de fases intermetálicas, que são danosas para as propriedades mecânicas e de resistência a corrosão dos AID. Portanto esse trabalho tem como objetivo fazer uma análise da região da ZAC de duas juntas de AID UNS 31803 soldadas pelo processo GMAW. A primeira junta foi soldada de acordo com a norma ISO 13847 , onde a temperatura interpasse máxima deve ser de $150^{\circ} \mathrm{C}$, desta forma na (junta A) a temperatura interpasse foi mantida abaixo de $150^{\circ} \mathrm{C}$. Na segunda junta os passes foram executados de forma simultânea sem tempo para resfriamento entre os passes, sendo que a temperatura interpasse se manteve entre $250^{\circ} \mathrm{C}-300^{\circ} \mathrm{C}$ (junta $\mathrm{B}$ ), conforme registrado com termômetro de contato. Como resultado, observou-se na junta B uma ZAC mais extensa e presença de fases intermetálicas com maior tamanho quando comparado à junta $\mathrm{A}$. Na realização da contagem de fases ferrita/austenita junto a zona afetada pelo calor (ZAC), foram encontrados para ambas as condições de soldagem uma porcentagem das fases semelhante, apresentando uma queda no percentual de austenita próxima a 10\% em comparação com o material base.

Palavras-chave: Aço inoxidável duplex; Processo GMAW pulsado; ZAC; Temperatura interpasse; Contagem de fases.

\footnotetext{
ABSTRACT

Duplex stainless steel (DSS) are materials with high mechanical strength and corrosion resistance in aggressive environments, the main requirements in chemical and petrochemical industry. These properties are assigned to its microstructural composition, which is around 50\% ferrite and 50\% austenite, based on balanced alloying elements and solubilization temperature. Generally, welding DSS applications involve thermal cycles, causing microstructure transformations. Therefore, welding processes must be properly controlled in order to reduce the occurrence of severe microstructural changes, as intermetallic phases, harmful to mechanical properties and corrosion resistance. The aim of this paper is to analyze a Heat Affected Zone (HAZ) on two DSS UNS 31803 welded joints resultant from GMAW process. The first one was welded according to
} 
standard ISO 13847 , with inter-pass temperature up to $150^{\circ} \mathrm{C}$ (joint A). The second one was welded through simultaneous passes, without cooling time, and inter-pass temperature range of $250^{\circ} \mathrm{C}-300^{\circ} \mathrm{C}$ (joint $\mathrm{B}$ ), measured with contact thermometer. HAZ and intermetallic phases were larger in joint $\mathrm{B}$ than in joint A. Phase counting ferrite/austenite $(\delta / \gamma)$ was carried out in the HAZ, and detected similar percentage for both welded conditions, comparing these results with base metal composition, there is a $10 \%$ reduction in austenite phase.

Keywords: Duplex stainless steel; Pulsed GMAW process; Inter-pass temperature, Phase counting.

\section{INTRODUÇÃO}

Aços inoxidáveis são materiais amplamente utilizados na atualidade devido às boas propriedades mecânicas e excelente resistência à corrosão. Dentro da família destes aços existe um grupo que possui essas propriedades ainda melhores, esses são chamados de aços inoxidáveis duplex (AID). Foram introduzidos na indústria por volta dos anos 70, a fim de suprir a demanda por materiais altamente resistentes para ambientes agressivos, como em plataformas offshore, na construção de pipelines, e na indústria química em contato com ácidos, entre outros. A razão pela qual os aços duplex possuem excelentes propriedades é devida principalmente ao seu balanço de fases entre ferrita e austenita, cerca de 50\% para cada fase. Porém é geralmente considerado que qualquer uma das duas fases não possua valores menores que $30 \%$ em volume, mantendo assim a característica de alta resistência mecânica da ferrita, com a ductilidade e resistência a corrosão da austenita 0 .

Os AID possuem alto nível de elementos de liga em relação aos austeníticos e ferríticos, principalmente a quantidade de nitrogênio, cromo e molibdênio. Essa grande quantidade de elementos de liga torna os AID muito suscetíveis à formação de fases intermetálicas devido à decomposição da fase ferrita quando submetido à procedimentos que geram um ciclo térmico como por exemplo a soldagem e tratamentos térmicos, requerendo-se um grande cuidado e planejamento no momento da realização de algum processo desta natureza 0 . Nos ciclos térmicos de soldagem tem-se a de exposição dos materiais a temperaturas propiciam a formação de fases intermetálicas. Após atingir temperaturas entre $300-1000^{\circ} \mathrm{C}$ os AID precipitam alguns intermetálicos como fase $\sigma$, fase $\alpha^{\prime}, \chi, \mathrm{CrN}, \mathrm{Cr}_{2} \mathrm{~N}$ e $\mathrm{M}_{7} \mathrm{C}_{3}$, as quais reduzem a ductilidade e principalmente a resistência a corrosão do material Erro! Fonte de referência não encontrada.. A mais prejudicial delas, a fase $\sigma$, cresce preferencialmente nos contornos de grão ferríticos e nas interfaces ferrita/austenita, crescendo na ferrita, devido ao alto teor concentrado de $\mathrm{Cr}$ e Mo existente na matriz. O crescimento da fase $\sigma$ na matriz ferrítica ocorre porque a ferrita é termodinamicamente instável na temperatura de formação da fase $\sigma$, estudos e trabalhos realizados comprovaram que isso se dá porque há um equilibro estrutural na temperatura de $650-900{ }^{\circ} \mathrm{C}$, e este equilíbrio é uma mistura de $\sigma$ e austenita. Este precipitado causa o empobrecimento do cromo na ferrita, pois intermetálicos ricos em cromo e molibdênio criam em seu redor zonas empobrecidas nesses elementos 0 . De acordo com vários autores, a precipitação da fase $\sigma$ pode ser retardada reduzindo os teores de cromo e molibdênio e aumentando os teores de níquel e nitrogênio. Porém diminuir o teor de Cr e Mo reduzirá também a resistência à corrosão por pite, corrosão por fresta e a resistência à tração, verificando-se assim que este não é o melhor método 0 . A formação da fase $\sigma$ também pode ser diminuída pela adição de níquel, porém é necessário um grande tempo de envelhecimento até o efeito de difusão do níquel com a formação de austenita. De acordo com Maehara et al. o Ni acelera a cinética de precipitação, contudo a fração de equilíbrio de $\sigma$ é reduzida. O mesmo não ocorre para o nitrogênio, que além de retardar a cinética de precipitação também aumenta a resistência à corrosão por cratera, sendo então a melhor alternativa para a desaceleração da precipitação da fase $\sigma$. O nitrogênio é um elemento gamagênico, que quando adicionado ajuda na estabilização da austenita e também gera aumentos das propriedades mecânicas e de resistência à corrosão por pite 0 . O motivo para isso está relacionado ao tamanho átomico do $\mathrm{N}$ muito reduzido, o que permite uma fácil difusão no estado sólido mesmo em temperaturas mais baixas, também devido à boa solubilidade na austenita o nitrogênio pode ser absorvido do gás de proteção, reduzindo assim as perdas desse elemento na ZAC, pois o nitrogênio tem tendência a migrar por difusão, do metal base para o metal de solda 0 .

Este trabalho tem como objetivo a análise microestrutural por meio de microscopia óptica e MEV, com foco na zona afetada pelo calor (ZAC), identificando a formação de fases intermetálicas, quando o aço inoxidável duplex UNS S31803 é submetido à soldagem multipasses respeitando-se o período de resfriamento entre os passes descrito em norma de no máximo de $150^{\circ} \mathrm{C} 0$, e quando a soldagem multipasses é feita de forma contínua, ou seja, sem período de resfriamento, utilizando-se para ambas as condições o gás de proteção $97,5 \%$ de $\operatorname{Ar}$ e $2,5 \%$ de $\mathrm{N}_{2}$. 


\section{MATERIAIS E MÉTODOS}

\subsection{Material base}

Este trabalho utilizou como material base (MB) um aço inoxidável duplex UNS S31803, na forma de chapa com espessura de $12,5 \mathrm{~mm}$, no estado solubilizado. Como metal de adição (MA) foi utilizado o eletrodo superduplex ER $2594 \mathrm{~L}$ de diâmetro 1,2 mm, garantindo assim o fornecimento do adequado conteúdo de elementos de liga durante o processo de soldagem. As tabelas 1 e 2 apresentam, de forma respectiva, as composições químicas do MB e MA e as propriedades mecânicas dos materiais utilizados.

Tabela 1: Composição química do MB e MA utilizados 0.

\begin{tabular}{c|c|c|c|c|c|c|c|c|cc}
\hline \multirow{2}{*}{ Material } & \multirow{2}{*}{ Classificação } & \multicolumn{6}{|c}{ Composição química (\% em peso). Fe balanço } \\
\cline { 3 - 11 } & & $\mathbf{C}$ & $\mathbf{C r}$ & Mo & Ni & N & Mn & Si & P & S \\
\hline \multirow{2}{*}{$\begin{array}{l}\text { MB } \\
\text { MA }\end{array}$} & UNS S 31803 & 0,016 & 21,84 & 2,74 & 4,95 & 0,225 & 1,6 & 0,36 & 0,011 & $<0,0010$ \\
\cline { 2 - 11 } & ER 2594 L & $<0,020$ & 25 & 4 & 9,5 & 0,25 & 0,4 & 0,3 & $<0,020$ & $<0,015$ \\
\hline
\end{tabular}

Tabela 2: Propriedades mecânicas do metal base (MB) e metal de adição (MA) 0.

\begin{tabular}{cccc}
\hline Material & $\begin{array}{c}\boldsymbol{\sigma}_{\mathbf{L E}} \\
(\mathbf{M P a})\end{array}$ & $\begin{array}{c}\boldsymbol{\sigma}_{\mathbf{L R}} \\
(\mathbf{M P a})\end{array}$ & $\begin{array}{c}\text { Alongamento } \\
(\mathbf{\%})\end{array}$ \\
\hline $\begin{array}{c}\text { Metal Base (MB) } \\
\text { Metal Adição } \\
(\mathrm{MA})\end{array}$ & 604 & 744 & 42 \\
\hline
\end{tabular}

\subsection{Parâmetros e equipamentos utilizados no processo de soldagem}

A geometria de junta adotada para a soldagem é apresentada na figura 2, onde foi utilizada uma junta com preparação meio $\mathrm{V}$ com ângulo de chanfro de $45^{\circ}$ e abertura de raiz de $2 \mathrm{~mm}$.

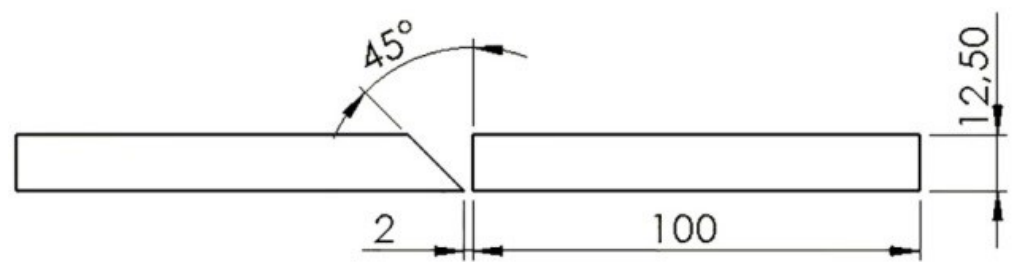

Figura 1: Geometria de junta utilizada no estudo.

A soldagem foi realizada por meio de um robô antropomórfico de 6 graus de liberdade modelo NB41NEFN com uma fonte de soldagem inversora modelo DP - 400. A soldagem foi efetuada em dois conjuntos denominados junta A e junta B, ocorreu na posição plana pelo processo GMAW pulsado, a maior capacidade de quebra de camada de óxidos gerada pela corrente pulsada justifica a sua aplicação na soldagem de aços duplex, a velocidade de soldagem foi de $5 \mathrm{~mm} / \mathrm{s}$, a corente de pico utilizada na soldagem foi de 250 A com tempo de pico de 1,5 s, a corrente de base foi 48 A com tempo de base 1,5 s sendo a corrente média do processo de 150 A e tensão média de $17 \mathrm{~V}$ a energia de soldagem para o processo foi de $0,510 \mathrm{~kJ} / \mathrm{mm}$. Durante o processo foi utilizada uma tocha com bocal de $18 \mathrm{~mm}$ de diâmetro para garantir a proteção da junta, distância bico de contato peça $18 \mathrm{~mm}$, para a proteção da poça de fusão foi utilizado gás $\mathrm{Ar}+2,5 \% \mathrm{~N}_{2}$ com vazão de $14 \mathrm{l} / \mathrm{min}$. A proteção da raiz foi realizada com gás de purga $100 \% \mathrm{~N}_{2}$ com vazão de $15 \mathrm{l} / \mathrm{min}$, o nitrogênio tem papel fundamental na estabilização da austenita junto a raiz da solda.

A temperatura entre passes foi monitorada durante o processo por termopar de contato marca Elcometer modelo 213 com intervalo de medição de -50 a $850^{\circ} \mathrm{C}$ com resolução de $1{ }^{\circ} \mathrm{C}$, o ponto de medição de temperaturas foi o centro do cordão depositado para as condições de soldagem estudadas. As juntas foram executadas 
em quatro passes, sendo um passe de raiz e três passes de enchimento executados de forma paralela.

\subsection{Análise metalográfica}

A análise metalográfica foi realizada utilizando microscópio ótico e microscópio eletrônico de varredura na ZAC das uniões. A preparação das amostras seguiu o processo padrão de preparação metalográfica com lixamento sequencial com lixas de granulometria na faixa de 220 a 1200, sendo o polimento realizado com solução de alumina $1 \mu \mathrm{m}$. O reagente químico utilizado foi o Behara Modificado com tempo de exposição entre 10 e 30 segundos, este procedimento foi utilizado para análise em MO e MEV.

Foram utilizados os seguintes microscópios para análise microscópio ótico marca ZEISS modelo Scope A1 e microscópio eletrônico de varredura marca Tescan modelo LM3. Com as imagens metalográficas em ampliações de $500 \mathrm{X}$ foi realizada uma contagem de fases $\delta / \gamma$ no software IMAGE J®.

\section{RESULTADOS}

\subsection{Aspectos superficiais das juntas soldadas}

A Figura 3 apresenta uma imagem da superfície dos cordões depositados, sendo na Figura 3(A) apresentada a junta A e na Figura 3(B) apresentada a junta B.

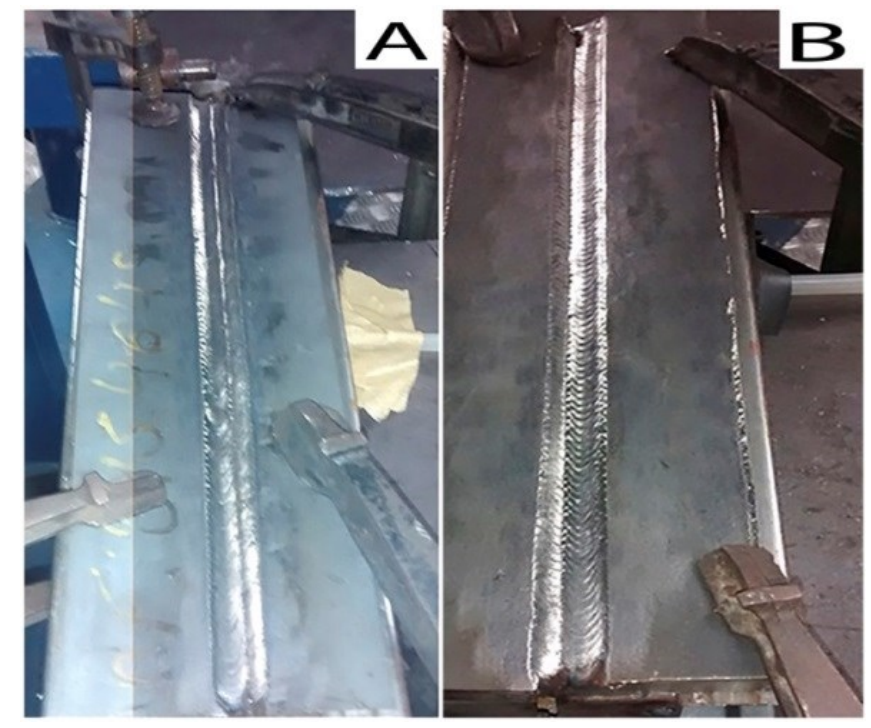

Figura 2: Em (A) Vista superior da junta A, em (B) a vista superior da junta B.

\subsection{Microscopia óptica}

\subsubsection{Análise metalográfica da junta soldada $A$}

Nas Figuras 4 (A e B) é possível visualizar a interface de soldagem da junta A sendo verificada modificações microestruturais quando comparada as regiões da ZAC e MA. Nas Figuras 4 (C, D) é possível visualizar modificações na morfologia da austenita com relação ao material de base solubilizado. 

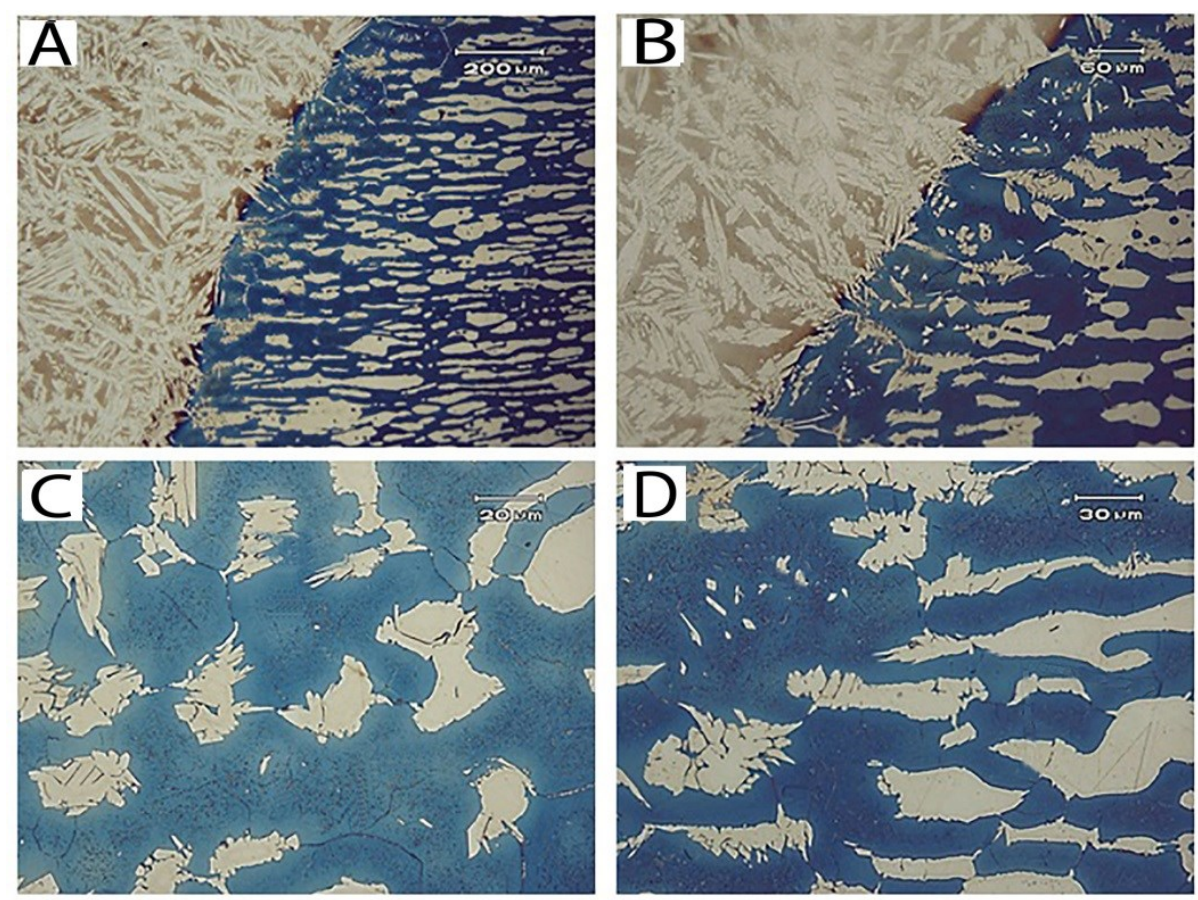

Figura 3: (A) Micrografia da região da ZAC junta A. (B) Micrografia da ZAC revelando intermetálicos. (C) ZAC (D) ZAC.

\subsubsection{Análise metalográfica da junta soldada B}

$\mathrm{Na}$ Figura 5 A é possível visualizar as alterações microestruturais ocorridas entre a ZAC e MA. As Figuras 5 $(\mathrm{B}, \mathrm{C})$ apresentam a microestrutura presente na $\mathrm{ZAC}$ da solda $\mathrm{B}$, sendo verificada modificações na morfologia da fase austenita nesta região.

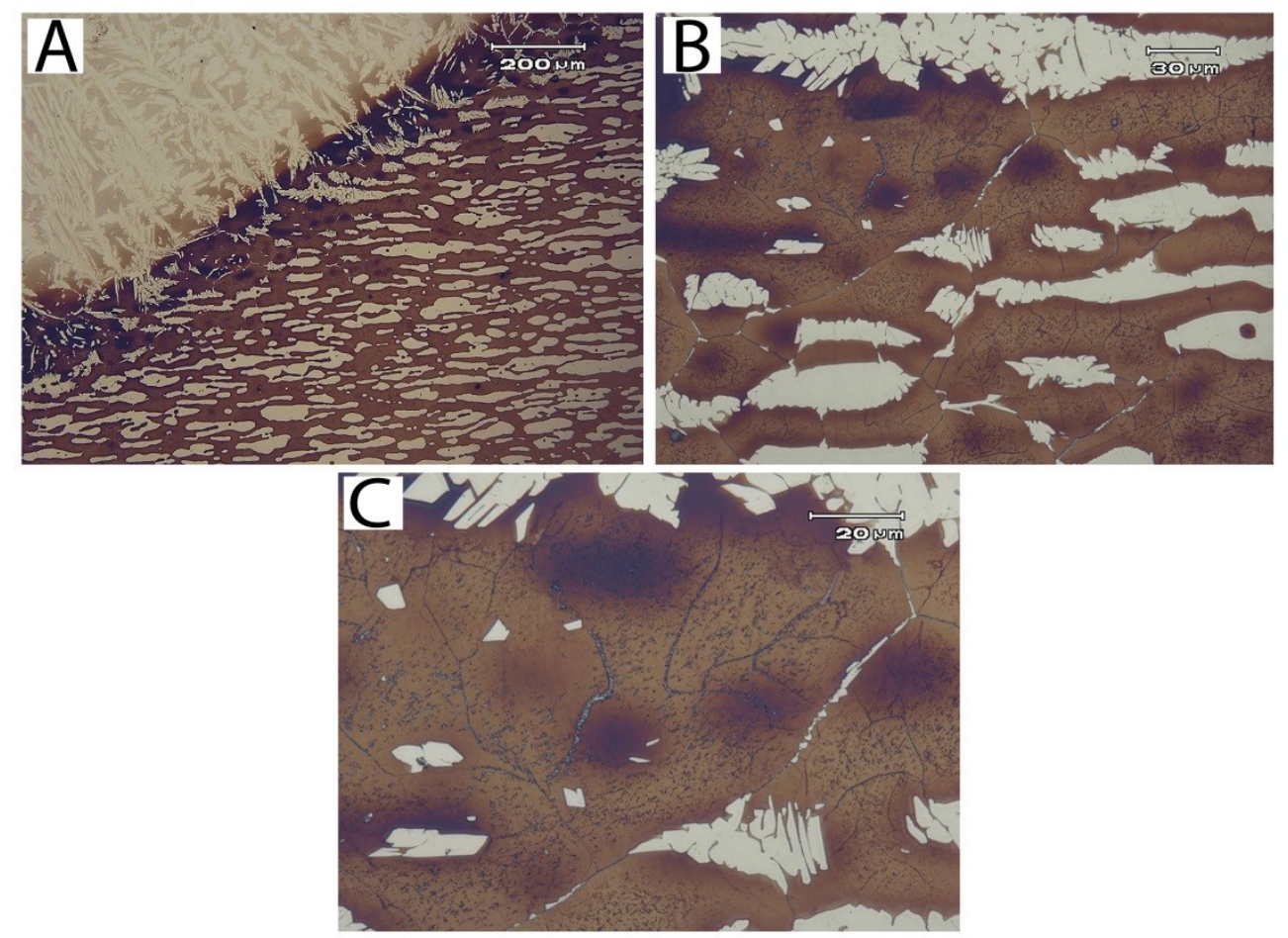

Figura 4: (A) Micrografia da região da ZAC junta B. (B) ZAC. (C) ZAC. 


\subsection{Análise em microscopia eletrônica de varredura (MEV)}

\subsubsection{Junta soldada $A$}

A Figura 6(A) é apresentada a microestrutura da interface de soldagem para a junta soldada A, as imagens apresentam a formação de precipitados em forma de bastões dispersos na ferrita morfologia semelhante a nitretos de cromo $\mathrm{Cr}_{2} \mathrm{~N}$ [1]. Os mesmos apresentam tamanho reduzido e uma pequena quantidade nucleando no contorno de grão ferritico. A Figura 6(C) com ampliação maior ampliação na fase ferrita apresenta precipitados com formato de pequenos bastões dispersos.
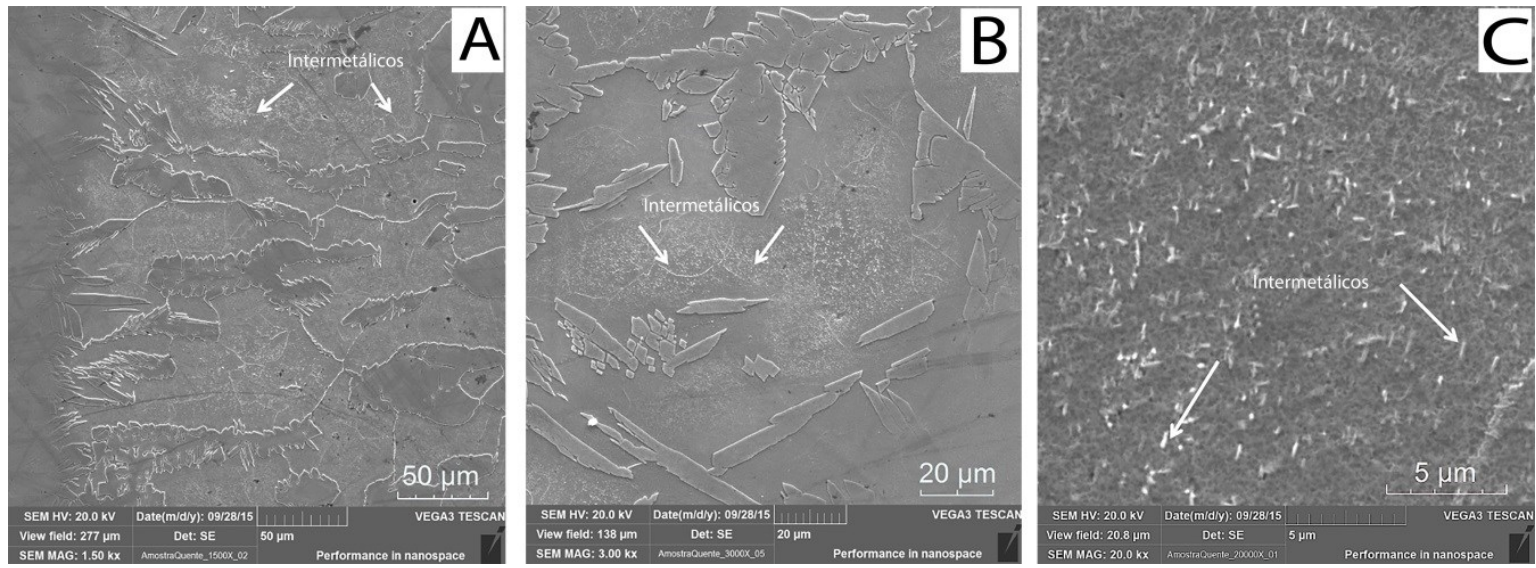

Figura 5: (A) Região interface MS/ZAC. (B) ZAC intermetálicos. (C) ZAC intermetálicos.

\subsubsection{Junta soldada B}

Na Figura 7(A) é apresentada a microestrutura da interface de soldagem para a junta soldada B, as Imagens 7(B) e 7(C) apresentam a microestrutura da ZAC com maior ampliação evidenciando a presença de fases intermetálicas dispersas na fase ferrita, estas apresentam morfologia de pontos dispersos com formato semelhante a nitretos de cromo $\mathrm{Cr}_{2} \mathrm{~N}$ e linhas de precipitados junto aos contornos de grão ferritico com morfologia compatível a nitretos de cromo $\mathrm{Cr}_{2} \mathrm{~N}$ estes intermetálicos ocorrem em maior tamanho e quantidade em comparação a junta A.
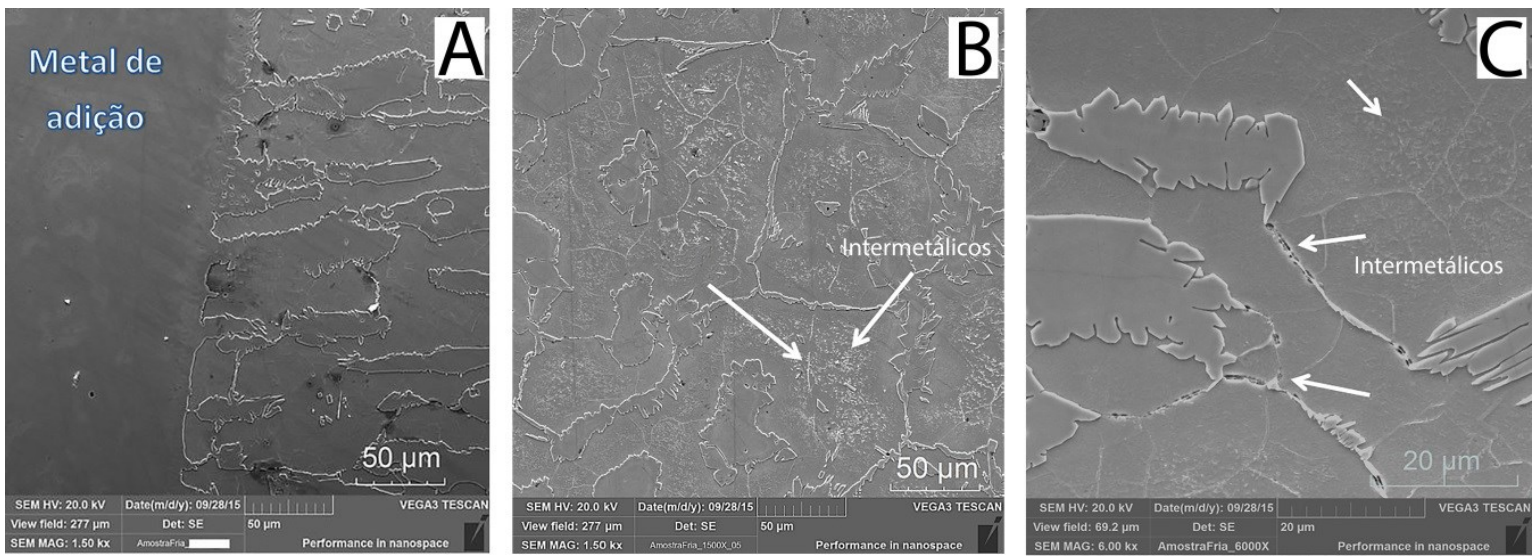

Figura 6: (A) Região interface MS/ZAC. (B) ZAC intermetálicos. (C) ZAC intermetálicos. 


\subsection{Contagem de fases}

A Figura 8 apresenta as imagens das micrografias realizadas e editadas no software IMAGE J® para contagem de fases segundo a norma ASTM E 562, em 8(A) está apresentado o balanço de fases para o metal base, em 8(B) a ZAC da junta A, e em 8(C) a junta B. Nas imagens as camadas em preto representam a austenita e o fundo branco representa a ferrita. A Figura 9 apresenta as porcentagens de ferrita e austenita obtidas em cada condição avaliada.

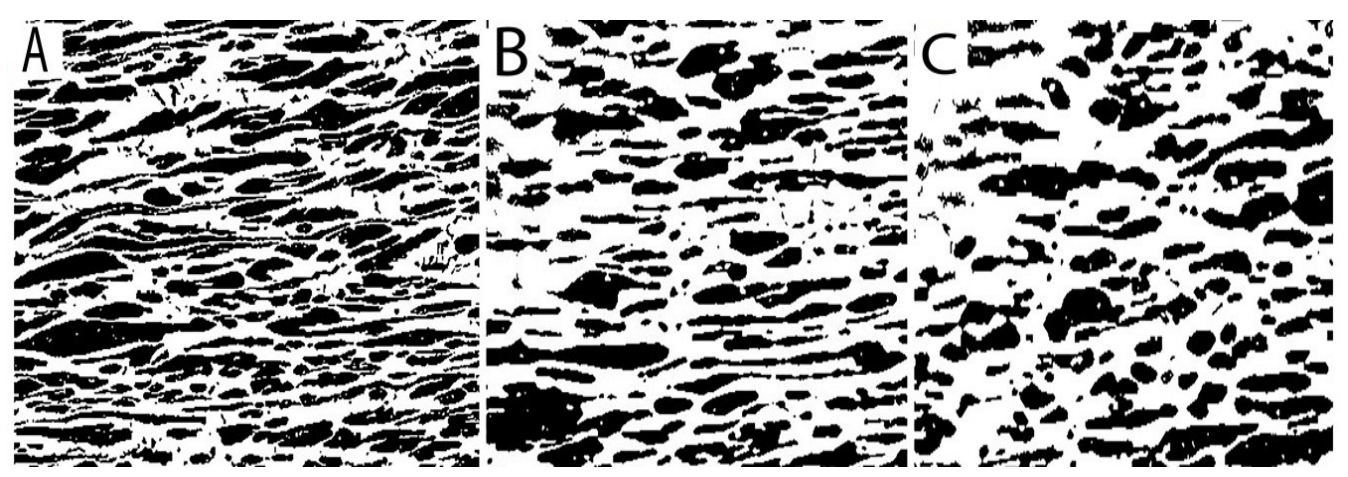

Figura 7: Imagens do balanço de fases da microestrutura software IMAGE J.

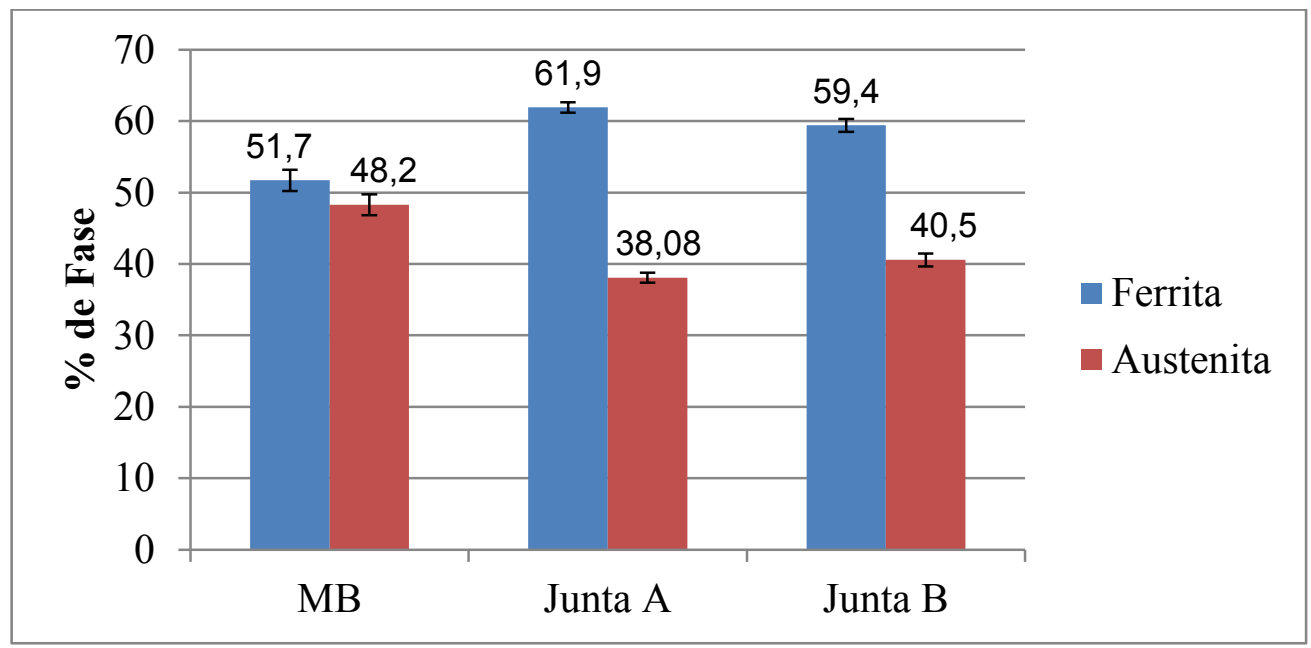

Figura 8: Resultado da contagem de fases.

\section{DISCUSSÃO}

Nas imagens de microscopia óptica verifica-se uma maior presença de intermetálicos na junta $B$, tendência que pode ser comparada analisando as Figuras $4(\mathrm{C})$ e 5(C). A maior formação dos intermetálicos bem como as suas maiores proporções de tamanho, ocorreram devido ao maior tempo de exposição à alta temperatura ter sido maior na junta soldada $\mathrm{B}$, tempo este é o responsável por permitir a nucleação de maiores quantidades de precipitados e permitir o seu crescimento 0

Sob análise de MEV também fica claro a maior presença e tamanho dos intermetálicos na junta soldada B, como pode ser visto na Figura 7(B) a presença de fase precipitadas com maior tamanho que as presentes na Figura 6(A) da junta A. Na imagem 6C com maior ampliação é possível verificar a presença de precipitados na forma de pequenos bastões, mostrando de acordo com a literatura técnica uma morfologia característica de nitretos de cromo 0 . A formação destes nitretos é explicada no processo de resfriamento a partir do campo ferritico, pois ocorre uma competição entre a precipitação de austenita e a de nitreto de cromo, se a quantidade de austenita formada for próxima ao valor de equilíbrio com a ferrita, toda a porcentagem de nitrogênio estará dissolvido nela, então a quantidade de precipitados de nitreto é praticamente nula. Por outro lado se a precipitação da austenita for retardada ou impedida principalmente por baixas energias de soldagem $(\mathrm{E}<0,5 \mathrm{~kJ} / \mathrm{mm})$ geram elevadas velocidades de resfriamento e facilitam a formação de elevadas frações de fase ferrita, sendo que a mesma torna-se supersaturada em nitrogênio, precipitando nitreto de cromo 0 . Ele- 
vadas temperaturas entre os passes de soldagem facilitam a nucleação e crescimento de precipitados durante a soldagem, este fator permitiu a nucleação e o crescimento de precipitados em maior quantidade na junta $\mathrm{B}$.

A cinética de precipitação para condição de soldagem é facilitada para formação de nitretos de cromo $\left(\mathrm{Cr}_{2} \mathrm{~N}\right)$ principalmente pelos baixos tempos de resfriamento, dificultando a nucleação das fases chi e sigma que necessitariam de maiores tempos em elevadas temperaturas para a sua formação.

$\mathrm{Na}$ contagem de fases verifica-se que ambas as condições de soldagem, apresentaram valores percentuais muito próximos de ferrita e austenita na região da ZAC apresentando um equilíbrio da quantidade das fases para as duas condições. Os resultados mostraram uma queda no percentual de austenita de $10,55 \%$ na ZAC da junta A quando comparado ao material base. A junta B apresentou uma queda percentual de austenita na ZAC de $9,77 \%$ quando comparado ao material base.

\section{CONCLUSÕES}

O tamanho da ZAC com modificações microestruturais foi maior para a condição de soldagem B devido a não se aguardar o período de resfriamento entre os passes, o que permitiu modificações microestruturais em uma região mais extensa da junta $\mathrm{B}$.

A formação de fases intermetálicas ocorreu em maior quantidade e com tamanho maior para a condição de soldagem B, sendo este crescimento de precipitados proveniente do maior tempo exposto a elevadas temperaturas, possibilitando o crescimento de fases intermetálicas.

A precipitação de nitreto de cromo $\left(\mathrm{Cr}_{2} \mathrm{~N}\right)$ foi facilitada para as condições de soldagem sendo que sua morfologia e distribuição foram diferentes para as duas condições de soldagem, evidenciando diferentes ciclos térmicos que proporcionaram diferentes cinéticas de precipitação.

A proporção de fases $\gamma$ e $\alpha$ junto a ZAC foi aproximadamente igual para as duas condições de soldagem ficando em aproximadamente $60 \%$ de ferrita, apresentando uma redução de aproximadamente $10 \%$ no percentual de austenita na ZAC das juntas A e B em comparação com os valores da fase austenita presentes no material base.

\section{AGRADECIMENTOS}

Os autores agradecem ao Laborátorio de Soldagem da Universidade de Passo Fundo (UPF) e ao Laboratório de Metalurgia Física- LAMEF- UFRGS, pelos equipamentos e materiais cedidos ao estudo, tornando possível este trabalho.

\section{BIBLIOGRAFIA}

[1] ROBERT, G. Duplex stainless steel- Microstructures, properties and applications, 1 ed.,Woodlhead Publishing, 1997.

[2] LIPPOLD, J.C., AL-RUMAIH, A.M. "Toughness and pitting corrosion of duplex stainless steel weld heat-affected zone microstructures containing secondary austenite", In: Conference Duplex Stainless Steels'97, pp.1005-1010, Maastrisht- The Netherlands, 1997.

[3] SOLOMON, H.D., DEVINE, T.M. Duplex stainless steel- a tale of two phases. In: Conference of Stainless Steel 1994. Proceedings, pp. 693-757, Ohio, 1894.

[4] BATERI, M., MANCIA, F., TAMBA, A.,et al., Microstructural Study and Corrosion Performance of Duplex and Superaustenitic Steels in Sour Well Environment, Corrosion: The journal of science and engeneering, v.43, n. 9, 1987.

[5] CHARLES, J. "Super duplex stainless steel: structure and properties", In: ConferenceProceedings Duplex Stainless Steel'91, Beaune, França, v.1, pp. 3, 1991.

[6] INTERNATIONAL MOLYBDENIUM ASSOCIATION - IMOA. Practical guidelines for the fabrication of duplex stainless steels, 1ed., London, England, 2001.

[7] ROSCOE, C.V., GRADWELL, K.J., LORIMER, G.W. "Struture/properties relationships in a series of duplex stainless steels", In: Duplex Stainless Steel 1984, pp. 563-777, Goteborg,Sweden, 1984.

[8] X.G. WANG, D. DUMORTIER, Y. RIQUIOR. Duplex Stainless Steel'91. Les editions de physique les Ulis, France, 1991.

[9] RADEMAKERS, P.L.F., VUIK, J., "Factors influencing microstructure and properties of weldments in duplex stainless steel”, In: Conference joining/welding 2000, pp.254-270, The Hauge- The Netherlands, 1991.

[10] GEIPL, H., "Gas mixture MAG welding corrosion-resistant duplex steels- Effect of shielding gas and 
process variations", In: Conference Duplex Stainless Steel 1986, pp.236-243, The Hauge- The Netherlands, 1986.

[11] FLORREN, S., H.D., HAYDEN. "The influence of austenite and ferrite on the mechanical properties of two phases stainless steel having microduplex structure”, Trans ASM, v.61, pp. 489-99, 1968.

[12] Maehara, Y,. Ohmori, Y,. Murayama, J., et al., "Effects of alloying elements on $\sigma$ phase precipitation in $\delta-\gamma$ duplex phase stainless steels", Metal Science, v.17, n.11, 1983.

[13] KOTECKI, D.J., "Ferrite control in duplex stainless steel weld metal”, Welding Journal, v.65, n.10, pp.273-278, 1986.

[14] LONDOÑO, A.J.R., BRANDI. S.D., "Estudo da adição de nitrogênio no gás de proteção na soldagem de aço inoxidável duplex UNS S31803”, In: XXII Encontro Nacional de Tecnologia da Soldagem, pp.277287, Blumenau, Brasil, 1996.

[15] FOLKARD, E., Welding Metalurgy of Stainless Steel, 1ed.,New York, Springer-Verlag, 1988.

[16] BAESLACK, W., LIPPOLD, J.C., "Phase transformation behavior in duplex stainless steels weldments", Metal construction, v.20,n.1, pp. 26-31,1988.

[17] DNV-OS-F101, Submarine Pipelines Systems, outubro 2010.

[18] ISO 13847, Petroleum and gas natural industries- Pipeline transportation systems- Welding of pipelies, 2013.

[19] POHL, M. “The Ferrite/Austenite Ratio of Duplex Stainless Steels”, Zeitschrift für Metallkunde, v.86, n.2, pp.97-102, 1995.

[20] Composição química duplex UNS31803, APERAM,

http://www.aperam.com/brasil/port/arquivos/DuplexUNS3220531803.pdf. Acessado em 20 de outubro de 2015.

[21] Composição química ER 2594. SANDVIK, http://smt.sandvik.com/en/materials-center/materialdatasheets/welding-products/welding-wire/sandvik-251041/. Acessado em 23 de outubro de 2015.

[22] ROLDÃO, A.M.B. Estudo do efeito do aporte térmico nas propriedades mecânicas e microestruturais do Aço Inoxidável Duplex UNS S 31803 em chapa grossa, soldado pelo processo GMAW, Tese de D. Sc., Universidade Federal de Minas Gerais, Belo Horizonte, MG, 2010.

[23] FONSECA, C. S., PINHEIRO, I.P., SILVA, S.N. Influência do aporte térmico sobre a morfologia da austenita e na quantidade das fases em chapas soldadas de aço inoxidável duplex SAF2205, Matéria, v.21, n.1, pp. 227-234, 2016. 